\title{
Modulation of Cortical Network Activity by Transcranial Alternating Current Stimulation
}

\author{
Randolph F. Helfrich and Till R. Schneider \\ Department of Neurophysiology and Pathophysiology, University Medical Center Hamburg-Eppendorf, 20246 Hamburg, Germany \\ Review of Ali et al.
}

In recent years, it has become evident that neuronal oscillations play a key role in human perception and behavior. In particular, synchronized activity in large-scale neuronal networks has been linked to different perceptual properties (Siegel et al., 2012). The ability to directly drive specific cortical rhythms offers a unique opportunity to reproduce functionally relevant oscillatory signatures and study subsequent behavioral performance (Thut et al., 2012). This approach could further extend the vast amount of correlative evidence and establish a causal link between brain oscillations and distinct cognitive functions.

To date, three methods for external neuronal entrainment are available: rhythmic sensory stimulation, rhythmic transcranial magnetic stimulation (rTMS), and transcranial alternating current stimulation (tACS). tACS might be a particularly powerful tool, because unlike rTMS it allows continuous stimulation below action-potential threshold without inducing a concomitant phase reset (Thut et al., 2011). In addition, tACS parameters, such as frequency or amplitude, can easily be varied and stimulation intensity can be kept below

Received Sept. 1, 2013; revised Sept. 27, 2013; accepted Oct. 8, 2013.

This work was supported by the German Research Foundation (DFG SFB936/Z1). R.F.H. is supported by the German National Academic Foundation. We thank lain Stitt for helpful discussions.

Correspondenceshould be addressed to Randolph F. Helfrich, Department of Neurophysiology and Pathophysiology, University Medical Center HamburgEppendorf, 20246 Hamburg, Germany. E-mail: r.helfrich@uke.de

DOI:10.1523/JNEUROSCI.3740-13.2013

Copyright $\odot 2013$ the authors $\quad 0270-6474 / 13 / 3317551-02 \$ 15.00 / 0$ skin sensation threshold. The physiological mechanisms of tACS are, however, poorly understood, because of excessive electrical stimulation artifacts in electrophysiological recordings (for review, see Herrmann et al., 2013). In the absence of compelling physiological evidence for neuronal entrainment by tACS, modeling approaches can be beneficial.

In a recent report, Ali et al. (2013) simulated large-scale networks of spiking neurons to investigate how weak global perturbations with alternating currents modulate the dynamics of neuronal oscillatory networks. They then confirmed simulation results by recording multiunit activity in anesthetized ferrets. The initial simulation supports the hypothesis that tACS can entrain neuronal networks in a frequency-specific manner through network resonance (Pikovsky, 2003; Herrmann et al., 2013). Resonance frequencies are stimulation frequencies at which weak periodic perturbations are particularly effective in entraining the network. Here, the authors demonstrated that a neuronal network with an intrinsic frequency of $\sim 3$ $\mathrm{Hz}$ can successfully be entrained by weak alternating currents of varying strength and frequencies. Crucially, the network was maximally entrained by external stimulation at its intrinsic frequency, but it was also increasingly excitable by stronger currents at adjacent frequencies. This dependency between current strength and stimulation frequency led to a conic synchronization area: the range of stimulation frequencies that successfully entrained the network broadened with higher stimulation currents, a phenomenon termed Arnold tongue (Ali et al., 2013, their Fig. 9A). In addition, the authors demonstrated that stimulation at or close to the first harmonic of the intrinsic network frequency was also powerful in entraining the network. Interestingly, the results revealed a suppression of neuronal activity at frequencies centered between the stimulation frequency and its first harmonic. The findings also demonstrate that the dominating frequency of the entrained network is shifted toward the stimulation frequency and away from the intrinsic frequency $(3 \mathrm{~Hz})$ when stimulation is applied at adjacent frequencies (e.g., 2.5 or 3.5 Hz; Ali et al., 2013, their Fig. 10).

As noted above, electrophysiological evidence for entrainment in vivo is limited to data from recordings made before and after stimulation because massive artifacts dominate recordings during transcranial electric stimulation. Ali et al. (2013) therefore pioneered a novel approach to study the rhythmic modulation of multiunit activity. Two ferrets were stimulated at different frequencies through two $2 \mathrm{~cm}^{2}$ electrodes attached bilaterally to the skull. Current intensity was set to $2 \mathrm{~mA}$ during all stimulation conditions. The obtained signals were high-pass filtered at $300 \mathrm{~Hz}$ and a threshold of -3 SDs was applied to extract the spike train. To analyze the discrete spiking activity by means of a continuous timefrequency analysis, the authors smoothed the spike train with a Gaussian kernel. The results indicated that rhythmic spiking activity during anesthesia could be driven 
by the externally applied stimulation, mainly in superficial cortical layers (Ali et al., 2013, their Fig. 11).

Although Ali et al. (2013) were able to demonstrate that alternating current stimulation elicited rhythmic spiking activity, the fundamental goal of tACS is to modulate ongoing brain activity with subthreshold currents to precipitate states of promoted or constrained neuronal excitability (Herrmann et al., 2013). However, compared with human EEG studies, an intensity of $2 \mathrm{~mA}$ through $2 \mathrm{~cm}^{2}$ electrodes is probably not subthreshold for action potentials: recent modeling of current flow revealed that $1 \mathrm{~mA}$ applied through $35 \mathrm{~cm}^{2}$ electrodes leads to an electric field of $\sim 0.4 \mathrm{~V} / \mathrm{m}$ (Herrmann et al., 2013). Animal studies have indicated that $>0.5 \mathrm{~V} / \mathrm{m}$ are necessary to elicit neuronal spiking activity. Therefore, transcranial stimulation with an intensity of 2 $\mathrm{mA}$ can be assumed to be above threshold for action potentials, especially when applied through smaller electrodes and an agar-sealed skull, which additionally facilitates the current flow.

In line with the neuronal entrainment hypothesis (Thut et al., 2012; Herrmann et al., 2013), experimental evidence has accumulated that ongoing cortical oscillations may become phase-locked to the external driving force (Neuling et al., 2013). Because phase is a free parameter in selfsustained systems (Pikovsky, 2003), enhanced oscillatory power might reflect synchronized neuronal activity, as has been observed after stimulation offset in human tACS experiments (Zaehle et al., 2010; Neuling et al., 2013). In the study by Ali et al. (2013), no intrinsic peak frequency before stimulation was reported and outlasting effects after stimulation were absent. Thus, it remains unclear whether the frequency-specific power increase was caused by an entrained oscillation or was instead related to rhythmically evoked spiking activity. Importantly, entrainment and network resonance would require synchronization and a direct interaction between driving force and underlying oscillatory activity (for a set of criteria, see Thut et al., 2011).Together, the results by Ali et al. (2013) demonstrate that neuronal spiking activity can be elicited through alternating electrical stimulation in a frequency-specific manner, but without additional evidence on phase interference it remains debatable whether the observed effects in the ferret experiment are related to network resonance phenomena.
The network simulation by Ali et al. (2013) with an average membrane depolarization of $<130 \mu \mathrm{V}$ is probably below stimulation intensities used in the ferret experiment; however, it is well within the range of human tACS studies. It is of particular interest that the network simulation revealed a prominent Arnold tongue linking stimulation frequency and stimulation intensity, because controversial findings on the frequency specificity of tACS were reported previously (Brignani et al., 2013). An unresolved question in human tACS experiments is which neuronal mechanism supports the increase in oscillatory power after stimulation offset (Zaehle et al., 2010; Neuling et al., 2013). Four mechanisms have been proposed: (1) phase alignment of different cortical oscillators (Thut et al., 2012; Neuling et al., 2013), (2) enhancement of endogenous power of single cortical oscillators, (3) recruitment of unengaged oscillators, and (4) mechanisms of neural plasticity (Zaehle et al., 2010). Therefore, network simulations are highly relevant to differentiate phase- from-amplitude-related effects (Zaehle et al., 2010; Ali et al., 2013). However, the absence of experimental findings supporting an Arnold tongue in tACS experiments (Moliadze et al., 2012) hampers the conclusions drawn by Ali et al. (2013). Moliadze and colleagues (2012) demonstrated that tACS effects relate to stimulation intensities in a nonlinear fashion: weak currents $(0.4 \mathrm{~mA})$ led to inhibition, higher intensities $(1 \mathrm{~mA})$ promoted excitation, and no effects were observed for intermediate intensities $(0.6$ and $0.8 \mathrm{~mA})$.

In summary, the exact physiological mechanism of tACS remains unknown and electrophysiological evidence is only starting to emerge. To gain an understanding of the physiological effects of tACS, a variety of parameters have to be assessed, foremost of which are electrode size and location, as well as stimulation frequency, intensity, and duration. Moreover, recent findings indicate that stimulation waveforms might also be important, since not only the absolute voltage levels determine neuronal discharge rates, but that steeper transient voltage changes influence neuronal firing (Fröhlich and McCormick, 2010). Ali et al. (2013) distinguish between hyperpolarizing-only (sinusoidal half-wave below zero) and depolarizing-only (sinusoidal half-wave above zero) stimulation (Ali et al., 2013, their Fig. $5 A$ ). The hyperpolarizing-only stimulation entrained the network as effectively as the fullwave stimulation, whereas the depolarizingonly stimulation entrained the network less effectively. Additionally, the network showed more regular dynamics under full-wave tACS and hyperpolarizing-only stimulation due to regular synaptic depression. The authors conclude that synaptic depression of excitatory synapses could recover in the hyperpolarizing phase of the stimulation leading to pronounced effects on neuronal oscillations.

In future, an elaboration of different parameters will provide more tailored stimulation approaches and enhance the physiological efficacy of tACS. Combined evidence from human, animal, and modeling studies will be highly beneficial to gain further insights into the dynamic interplay between ongoing brain activity and the externally applied sinusoidal stimulation.

\section{References}

Ali MM, Sellers KK, Fröhlich F (2013) Transcranial alternating current stimulation modulates large-scale cortical network activity by network resonance. J Neurosci 33:1126211275. CrossRef Medline

Brignani D, Ruzzoli M, Mauri P, Miniussi C (2013) Is transcranial alternating current stimulation effective in modulating brain oscillations? PloS One 8:e56589. CrossRef Medline

Fröhlich F, McCormick DA (2010) Endogenous electric fields may guide neocortical network activity. Neuron 67:129-143. CrossRef Medline

Herrmann C, Rach S, Neuling T, Strüber D (2013) Transcranial alternating current stimulation: a review of the underlying mechanisms and modulation of cognitive processes. Front Hum Neurosci 7:279. CrossRef Medline

Moliadze V, Atalay D, Antal A, Paulus W (2012) Close to threshold transcranial electrical stimulation preferentially activates inhibitory networks before switching to excitation with higher intensities. Brain Stimul 5:505-511. CrossRef Medline

Neuling T, Rach S, Herrmann CS (2013) Orchestrating neuronal networks: sustained after-effects of transcranial alternating current stimulation depend upon brain states. Front Hum Neurosci 7:161. CrossRef Medline

Pikovsky A (2003) Synchronization: a universal concept in nonlinear sciences. Cambridge, UK: Cambridge UP.

Siegel M, Donner TH, Engel AK (2012) Spectral fingerprints of large-scale neuronal interactions. Nat Rev Neurosci 13:121-134. CrossRef Medline

Thut G, Schyns PG, Gross J (2011) Entrainment of perceptually relevant brain oscillations by non-invasive rhythmic stimulation of the human brain. Front Psychol 2:170. CrossRef Medline

Thut G, Miniussi C, Gross J (2012) The functional importance of rhythmic activity in the brain. Curr Biol 22:R658-R663. CrossRef

Zaehle T, Rach S, Herrmann CS (2010) Transcranial alternating current stimulation enhances individual alpha activity in human EEG. PloS One 5:e13766. CrossRef Medline 